\title{
REVIEW
}

\section{Recommendations for mental health professionals in the NICU}

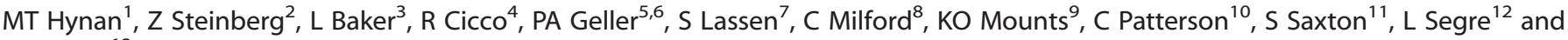 \\ A Stuebe ${ }^{13}$
}

This article describes recommended activities of social workers, psychologists and psychiatric staff within the neonatal intensive care unit (NICU). NICU mental health professionals (NMHPs) should interact with all NICU parents in providing emotional support, screening, education, psychotherapy and teleservices for families. NMHPs should also offer educational and emotional support for the NICU health-care staff. NMHPs should function at all levels of layered care delivered to NICU parents. Methods of screening for emotional distress are described, as well as evidence for the benefits of peer-to-peer support and psychotherapy delivered in the NICU. In the ideal NICU, care for the emotional and educational needs of NICU parents are outcomes equal in importance to the health and development of their babies. Whenever possible, NMHPs should be involved with parents from the antepartum period through after discharge.

Journal of Perinatology (2015) 35, S14-S18; doi:10.1038/jp.2015.144

\section{BACKGROUND}

Numerous research studies have documented elevated levels of emotional distress in parents during the hospitalization of their baby in a neonatal intensive care unit (NICU) and thereafter. ${ }^{1-5}$ Many of these studies have reported clinically elevated levels of postpartum depression (PPD) and post-traumatic stress disorder (PTSD). Although not all NICU parents have these disorders, it has been estimated from research in the NICU that 20 to $30 \%$ or higher of NICU parents experience a diagnosable mental disorder during the first postpartum year. ${ }^{6}$ An additional proportion of NICU parents will experience subclinical levels of symptoms. These symptoms have many harmful correlates, including: (a) interference with parental trips to the NICU, (b) disturbances in both mothers' and fathers' developing relationship with their baby and (c) later impairments in the growth and development of their baby and child. ${ }^{7-16}$

Layered levels of emotional support should be available to all parents in the NICU, ${ }^{17}$ including systematic support and education of parents in the developmental needs of NICU babies (see 'Recommendations for involving the family in developmental care of the NICU baby', this issue). Peer-to-peer support provided by trained volunteers should also be offered to all parents, with in-person support as a best practice (see 'Recommendations for peer-to-peer support for NICU parents', this issue). This combination constitutes a 'universal' level of care for all parents. ${ }^{17}$ For parents with more risk factors and/or those displaying acute distress, NICUs should provide support from social workers, psychologists, psychiatrists, pastoral care staff and NICU staff trained as paraprofessional counselors (i.e., 'targeted care'). ${ }^{17}$ NICUs should also have referral mechanisms in place for psychological and psychiatric treatment outside the NICU for those parents whose symptoms require 'clinical' ${ }^{17}$ levels of care beyond the capabilities of the NICU. Whenever possible, support should begin in the antepartum period and be carried through the after discharge period (see 'NICU discharge planning and beyond: recommendations for parent psychosocial support,' this issue).

The following are recommended best practices for supporting the emotional well-being of parents while in the NICU and preventing a deterioration of psychological functioning during the potentially traumatic NICU experience. ${ }^{18,19}$

\section{RECOMMENDATIONS FOR NICU MENTAL HEALTH PROFESSIONALS}

1. All NICUs with 20 or more beds shall have at least one full-time masters' level social worker and one full-time or part-time doctoral level psychologist embedded in the NICU staff. NICUs should also consider having full-time or part-time psychiatrists and psychiatric nurses on staff. Larger NICUs should have proportionally more NICU mental health professionals (NMHPs) on staff. There is a growing trend toward employing psychologists in NICUs, and some NICUs have had psychologists on staff for decades (http://www.nationalperinatal.org/psychologists). Funding streams for NMHPs, including third-party reimbursement, vary state by state. NICUs are encouraged to investigate sources of funding for mental health services with

${ }^{1}$ Department of Psychology, University of Wisconsin-Milwaukee, Milwaukee, WI, USA; ${ }^{2}$ Department of Pediatrics, Division of Neonatology, New York Presbyterian Morgan Stanley Children's Hospital, Columbia University, New York, NY, USA; ${ }^{3}$ Department of Social Work, University of Alabama at Birmingham, Birmingham, AL, USA; ${ }^{4}$ Department of Neonatology, West Penn Hospital, Pittsburgh, PA, USA; ${ }^{5}$ Department of Psychology, Drexel University, Philadelphia, PA, USA; ${ }^{6}$ Department of Obstetrics and Gynecology, Drexel University College of Medicine, Philadelphia, PA, USA; ${ }^{7}$ Department of Pediatrics, University of Kansas Medical Center, Kansas City, KS, USA; ${ }^{8}$ Cheryl Milford Consulting, Huntington Beach, CA, USA; ${ }^{9}$ Wisconsin Association for Perinatal Care/Perinatal Foundation, Madison, WI, USA; ${ }^{10}$ Division of Neonatology, Children's Hospital of Philadelphia, Philadelphia, PA, USA; ${ }^{11}$ Department of Pediatrics and Neonatology, Health and Science University, Portland, OR, USA; ${ }^{12}$ College of Nursing, and Department of Psychological and Brain Science, University of lowa, lowa City, IA, USA and ${ }^{13}$ Division of Maternal Fetal Medicine, University of North Carolina, Chapel Hill, NC, USA. Correspondence: Dr MT Hynan, 9836 Leeward Avenue, Key Largo, FL 33037, USA. 
their hospital administrators, specifically chief financial officers and medical billing professionals.

2. All NICUs should provide at least one comfortable area for group discussions among parents (e.g., parent lounge) and one comfortable room per 20 beds for confidential discussions between NICU families and NMHPs.

3. The 'Standards for Social Work Services in the NICU' of the National Association for Perinatal Social Workers (NAPSW) are endorsed, along with the NAPSW standards for social workers regarding PPD and perinatal bereavement (http://www.napsw. org/about/napsw-code-of-ethics-and-standards.html).

4. The roles of social work and psychology can overlap regarding activities such as counseling, screening, providing staff education and teaching parenting skills.

5. Procurement of benefits and services for families from outside agencies should continue to be one of the roles of social workers. Social workers, psychologists and psychiatric staff should have dedicated time to provide verbal therapeutic support to all parents and family members. NMHPs are essential members of the NICU care team and should interact with all NICU parents/caregivers and family members. Collaboration among social workers, psychologists and psychiatric staff is strongly encouraged to provide support to both parents and NICU staff. Some hospitals have shifted case management responsibilities to nurse case managers, so social workers can provide necessary support for both families and NICU staff.

6. Roles of psychologists, psychiatric staff or advanced clinical social workers (when within the scope of their credentials) can include:

a. Conducting research in the following areas:

i. Use of assessment devices, test interpretation and outcome evaluation.

ii. Identifying risk for the development of psychological disorders in NICU parents. ${ }^{6}$

iii. Parent-infant attachment and therapy.

iv. The effects of parental emotional distress on both the parent-child relationship, the parental couple and the longterm outcomes of the child (both physical and emotional).

b. Providing the following clinical services for families:

i. Assessments, test interpretation and outcome evaluation.

ii. Differential diagnoses of psychiatric disorders and recognition of subclinical symptoms.

iii. A variety of treatment approaches, including interpersonal therapy, short-term dynamic therapy, cognitive therapy, behavior therapy, couples and family therapy, mindfulness training and infant mental health.

c. Educating NICU staff about both the centrality of the parent dyad-infant relationship in all interactions and communicating with families who are: (a) guilt and shame-ridden, (b) distressed and angry, (c) possibly struggling with substance abuse, (d) bereaved and (e) coping with prior traumas and perinatal losses including multiple trials of assisted reproductive technology and miscarriages.

7. Social workers, psychiatric staff and psychologists who work in NICUs should provide support to staff as well as to families (see 'Recommendations for enhancing psychosocial support of NICU parents through staff education and support', this issue). Supporting roles include acting as liaisons between staff and families along with direct educational support by discussions of family dynamics and family-staff interactions. These discussions should occur in a variety of settings including rounds, case conferences, faculty meetings, debriefing sessions, and so on. Such support is necessary to minimize burnout, compassion fatigue and secondary traumatic stress. Pastoral care staff can also be instrumental in providing this support.

Clinical judgment of NMHPs is paramount in making decisions about implementing recommendations regarding screening, treatment and referral. These judgments should take into account the diversity of families' ethnic, religious and social relationships. Special circumstances may require adaptation of these recommendations.

NMHPs should endeavor to create a family-centered culture of understanding and responsiveness for the NICU staff (medical, administrative, custodial, volunteer, and so on) that recognizes, normalizes and supports NICU parental emotional distress. Ideally this culture should permeate the NICU staff and be readily apparent to NICU families. Within this culture, care for the emotional and educational needs of NICU parents are outcomes equal in importance to the health and development of their babies.

\section{RECOMMENDATIONS FOR LAYERED LEVELS OF SUPPORT FOR NICU PARENTS AND FAMILIES}

1. The family-centered NICU environment should include an active peer-to-peer support organization, ideally with a position for a paid parent support coordinator embedded in the NICU staff. NMHPs should work closely with the parent support coordinator providing training to recognize parental emotional distress and perinatal mood disorders.

2. All NMHPs should strive to meet with all parents/caregivers to screen and identify parents at high risk for emotional disorders and those with subclinical symptoms. Clinical judgment and a consideration of other risk factors should determine the outcome of a screen. When first meeting with parents and caregivers, social workers and psychologists should explicitly state that they meet and interact with 'all parents' so as to (a) normalize the likelihood of parental emotional distress, (b) avoid perceptions of stigmatization of distress and (c) initiate the process of providing emotional support. One goal of NMHP interactions with parents is to identify both: (a) parents with clinical levels of symptoms and (b) parents with subclinical symptoms, who are also at increased risk. A positive identification (or subclinical score) may not require referral to a mental health provider but should result in additional attention and targeted support while in the NICU. Increased levels of support can be given by embedded NMHPs as well as other NICU staff trained (a) to recognize symptoms of PPD, PTSD and other psychological problems and (b) to provide a first level of emotional support ${ }^{20}$ (e.g., listening visits). ${ }^{21,22}$

3. All NICUs with at least 40 beds should have parent education groups with a therapeutic orientation, which meet at least once a week. These groups should be led by NMHPs and should supplement any group meetings conducted by the parent-toparent group. ${ }^{23-25}$ Parent education and support groups should meet at flexible hours to avoid infant feeding hours and so that working parents and grandparents may attend. Some NICUs have found that alternating between an afternoon and an evening (or weekend) works well. Group meetings and topics should be advertised at bedside and on bulletin boards. NICU staff receiving electronic messages can also be prompted to invite parents to meetings. Groups should be facilitated by NMHPs (and ideally cofacilitated by other NICU staff or trained parent volunteers). Experienced NMHPs and peer-to-peer volunteers have reported that labeling parent meetings as only 'support' groups may diminish attendance because of stigmatization and/or parents' fear of exposure. Instead, announcing content, such as 'Learn to Read your Babies Cues', 'Breastfeeding-Pumping May Be a Drag, but...', 'Tips for Surviving the NICU' or 'Dads-Your Special Role', can help 
increase attendance (along with the provision of food). Additional suggestions for parent support can be found at an online 'tool kit' developed by members of the supplemental issue workgroup (www.support4NICUparents.org).

4. All NICUs should have resources within the NICU and affiliated hospital for caring for the $20-30 \%$ (or higher) of NICU parents likely to experience a diagnosable mental disorder. ${ }^{6}$ All NICUs should also have referral mechanisms in place for treatment outside of the hospital by social workers, psychologists, psychiatric nurses and psychiatrists. NICUs lacking a parent support organization or NICUs without NMHPs should ensure that referrals are available to all parents for emotional support. Possible resources include hospital parent advisory boards, community parent support organizations and outside referrals to mental health professionals. Research has indicated, however, that outside referrals are often not accomplished. ${ }^{26-28}$ Families are often too stressed and burdened to keep appointments at outside facilities. Counseling and therapy conducted in the NICU are more likely to be successful with parents attending more often, and thus likely to gain therapeutic benefit. Some NICUs, with established psychotherapeutic programs, have also arranged for psychotherapists from Behavioral Health units in the hospital to offer therapy in the NICU on an outpatient basis. Research on psychotherapy conducted in the NICU has shown beneficial results. ${ }^{29-32}$ Special attention needs to be paid to the parents that rarely come to the NICU. Although it might be that distances are too great and/or the burdens of other children are heavy, it also may be a symptom of significant depression and/or a traumatic reaction, as avoidance is a notable symptom of PTSD. Outreach needs to be done to engage these parents.

5. Using the NICU's standards of confidentiality, NMHPs should strive to communicate the identified mental health needs of parents/caregivers with the obstetric care provider and the family's primary care provider, pediatrician and other care providers.

\section{RECOMMENDATIONS FOR SCREENING IN THE NICU FOR EMOTIONAL DISTRESS}

1. NMHPs should strive to meet with all parents/primary caregivers within 1-3 days of admission to establish a working relationship, normalize emotional distress and evaluate risk factors for all forms of emotional distress. Hynan et al. ${ }^{6}$ reported a table of replicated predictors of elevated scores for PPD and PTSD. Whenever possible NMHPs should use standardized measures for evaluation. There are many validated screens for PPD and PTSD. ${ }^{6}$

2. Screening should be done within the first week (both mothers and fathers). ${ }^{6}$ Screening for emotional distress is best done early to evaluate parents whose babies are in the NICU for only a few days. Parents with shorter stays have reported elevated levels of emotional distress. ${ }^{4,5,33}$

3. NICU parent/caregivers should be rescreened later: (a) whenever deemed important and (b) within $48 \mathrm{~h}$ before discharge (for NICU stays $>1$ week). Because levels of emotional distress change (see 'Introduction: psychosocial program standards for NICU parents', this issue), NICU parent/caregivers should be rescreened later.

4. Screening methods can include any of the following (in order of complexity): ${ }^{6}$

a. quick screens for depression (2 questions) $)^{34}$ and PTSD (4 questions), ${ }^{35}$

b. validated PPD and PTSD questionnaires, ${ }^{36-43}$ c. inventories, such as the Psychological Assessment Tool$\mathrm{NICU}^{44}$ and

d. interviews, such as the Clinical Interview for Premature Parents. $^{13,45}$

5. Positive screens at a less complex level should be followed up with more complex screens. Choice of screening method is best left to the judgment of NMHPs, taking into consideration the general procedures in a particular NICU. Responsibility for administering less complex screens (a-c above) can be delegated to NICU staff, specifically those trained and supervised by an NMHP. Some NICUs have had success with having the bedside nurse administer screens. More complex interviews should by administered by NMHPs. In any case, it is advantageous to have the person administering the screen be someone who has developed a positive working relationship with the parents, often a bedside nurse. ${ }^{46}$ Simply leaving paper and pencil screens by the bedside is insufficient for adequate screening. NICU staff should be educated regarding the rationale for screening and typical follow-up. A single screen should be viewed as one piece of information, not a whole clinical picture. Screening by itself is a necessary, but insufficient, way of both meeting the mental health needs of families and evaluating the parent-infant relationship needs. (The Clinical Interview for Premature Parents interview is one method of exploring the parent-infant relationship.) $)^{13,45}$ Screening methods may need to be modified by concerns such as the education level of the parent, language, cultural tradition and so on. NICUs upgrading their psychosocial services for parents have faced questions of how to record information regarding screening results, therapy progress and referrals. These questions are especially relevant in children's hospitals when parents are not the patients. Using hospitalbased standards of confidentiality, we recommend that NICU staff and NMHPs make judgments on information storage, retrieval and communication that safeguards parents' confidentiality as much as possible while (a) maximizing services to parents in the NICU and (b) facilitating the emotional wellbeing of parents after discharge. Suggestions for recording this information, as well as the implementation of other NMHP recommendations, can be found on the online 'tool kit' developed by members of the supplemental issue workgroup (www.support4NICUparents.org).

6. Screening should be incorporated into the NICU procedures as quality assurance.

7. Screening should only be implemented if there is psychological treatment available either within the NICU or through outside referral. NICUs without NMHPs may still use screening if outside referral networks are in place. In this case, NICU staff should be trained by someone with expertise in psychometrics, test interpretation and referral. If an NICU is not able to provide embedded NMHP staff on a full-time basis, part-time staffing by mental health professionals from other units of the hospital is essential.

8. Screening for emotional distress should be offered to parents returning with their babies for developmental follow-up visits.

Although some families manifest symptoms of depression, anxiety and PTSD during the NICU hospitalization, others may experience symptoms after the baby is discharged or as parents recognize that their child is not achieving expected developmental milestones. Thus, parents should also be screened after discharge by health-care home visitors and at developmental follow-up appointments. During follow-up visits, NICU parents should be educated about the vulnerable child syndrome and other possible conditions, as well as screened for possible abuse. 


\section{RECOMMENDATIONS FOR TELEMEDICINE SUPPORT}

1. Telemedicine can be used for both screening ${ }^{47}$ and treatment, ${ }^{48-50}$ potentially increasing accessibility for families in low resource settings.

2. Telemedicine services shall be conducted in accordance with accepted standards for training, providers' credentials, confidentiality and HIPPA compliance, as developed (for example) by The American Telemedicine Association (http://www.amer icantelemed.org/resources/telemedicine-practice-guidelines/tel emedicine-practice-guidelines/clinical-guidelines-for-telepathol ogy\#.VOTXRth0ypo). Teletherapy and screening for NICU parents can follow the guidelines of the American Psychological Association (http://www.apapracticecentral.org/ce/guide lines/telepsychology-guidelines.pdf).

3. Many NICU parents use social media from Internet sites for both information and emotional support. NICU staff should familiarize themselves with web-based support sites to guide parents to reliable sources. ${ }^{51-56}$

Many NICU parents are unable to come into the NICU on a regular basis. Impediments include long travel distances, lack of transportation, poverty, need for uninterrupted employment, childcare and (often ignored) mental health issues. NICUs with access to adequate information technology support should establish telemedicine services for both NICU babies and parents. Research has indicated that outcomes using telemedicine services are often equivalent to outcomes from face-to-face contact. ${ }^{57,58}$

\section{RECOMMENDATIONS FOR ANTENATAL SCREENING AND SUPPORT}

1. When a NICU stay is anticipated, parent support coordinators and NMHPs should initiate a relationship with the family before the birth to provide both emotional support and prenatal screening for emotional distress. ${ }^{59}$

Among women admitted for antepartum pregnancy complications, the prevalence of anxiety and depression symptoms is high. ${ }^{60}$ Evidence suggests that women with antenatal depression and PTSD are at increased risk of preeclampsia and preterm birth. ${ }^{61-63}$

\section{SUMMARY}

Evidence-based NICU care has been evolving over the past few decades. Research has documented that NICU parents have a variety of emotional responses to the NICU experience, ranging from resilience to psychopathology. ${ }^{2,3,6,19}$ Three variables are likely responsible for much of this variability: pre-existing mental health conditions, the severity of the baby's medical condition and the level of emotional support for parents during the potentially traumatic NICU experience. ${ }^{18}$ The NICU staff can do little about the first variable, other than inquire about prior health conditions of parents and act accordingly. NICU care has historically been focused on the physical health of the baby, but the technological medical advances in the NICU may be approaching limits. Intensifying the focus in the NICU on the emotional well-being of parents is the next frontier. Evidence has also documented that this frontier has a great potential to (a) facilitate the growth and development of babies through a healthy parent-baby relationship and (b) promote the long-term emotional well-being of parents, babies and families. Movement towards this goal will also be aided by an NICU culture that values the emotional well-being of all staff, encouraged to work together in an interdisciplinary manner.

\section{CONFLICT OF INTEREST}

The authors declare no conflict of interest.

\section{ACKNOWLEDGEMENTS}

We are grateful to other members of the NICU Mental Health Professional workgroup for their contributions: Amy Baughcum, Susan Hatters Friedman, Becky Hatfield; Pec Indman, Carrie Piazza-Waggoner, Keira Sorrells; and Sarah Verbiest. This article is dedicated to an earlier generation of NICU staff and parents who showed great concern for NICU parent care: John Glaspey, Helen Harrison, August 'Larry' Jung, Sheri Nance and countless others. This article has been supported by grants and contributions from: the Wellness Network, Prolacta Bioscience, Loma Linda University Department of Pediatrics, NICU Parent Support at Mercy Hospital in St Louis, Missouri, Hand to Hold, Preemie Parent Alliance, Zoe Rose Memorial Foundation, the Rosemary Kennedy Trust and Eden's Garden.

\section{REFERENCES}

1 Friedman SH, Kessler A, Yang SN, Parsons S, Friedman H, Martin RJ. Delivering perinatal psychiatric services in the neonatal intensive care unit. Acta Paediatr 2013; 102: e392-e397.

2 Penny KA, Freidman SH, Halstead GM. Psychiatric support for mothers in the neonatal intensive care unit. J Perinatol 2015; 35(6): 451-457.

3 Holditch-Davis D, Miles MS, Weaver MA, Black B, Beeber L, Thoyre S et al. Patterns of distress in African-American mothers of preterm infants. J Dev Behav Pediatr 2009; 30(3): 193-205.

4 Brandon D, Tully K, Silva S, Malcolm W, Murtha A, Turner BS et al. Emotional responses of mothers of late-preterm and term infants. $J$ Obstet Gynecol Neonatal Nurs 2011; 40(6): 719-731.

5 Voegtline K, Stifter C, The Family Life Project Investigators Late-preterm birth, maternal symptomatology, and infant negativity. Infant Behav Dev 2010; 33(4): 545-554.

6 Hynan M, Mounts K, Vanderbilt D. Screening parents of high-risk infants for emotional distress: rationale and recommendations. J Perinatol 2013; 33(10): 748-753.

7 Diego $M$, Field $T$, Jones $N$, Hernandez-Reif $M$. Withdrawn and intrusive maternal interaction style and infant frontal EEG symmetry shifts in infants of depressed and non-depressed mothers. Infant Behav Dev 2006; 29(2): 220-229.

8 Murray L, Arteche A, Fearon P, Halligan S, Goodye I, Cooper P. Maternal postnatal depression and the development of depression in offspring up to 16 years of age. $J$ Am Acad Child Adolesc Psychiatry 2011; 50(5): 460-470.

9 Ramchandani P, Psychogiou L, Vlachos H, lles J, Sethna V, Netsi E et al. Paternal depression: an examination of its links with father, child and family functioning in the postnatal period. Depress Anxiety 2011; 28(6): 471-477.

10 Davis R, Davis M, Freed G, Clark S. Fathers' depression related to positive and negative parenting behaviors with 1-year old children. Pediatrics 2011; 127(4): 612-618.

11 McManus B, Poehlmann J. Maternal depression and perceived social support as predictors of cognitive function trajectories during the first 3 years of life for preterm infants in Wisconsin. Child Care Health Dev 2012; 38(3): 425-434.

12 Korja R, Savonlahti E, Haataja L, Lapinleimu H, Manninen H, Piha J et al. PIPARI Study Group. Attachment representations in mothers of preterm infants. Infant Behav Dev 2009; 32(3): 305-311.

13 Keren M, Feldman R, Eidelman Al, Sirota L, Lester B. Clinical interview for high-risk parents of premature infants (CLIP) as a predictor of early disruptions in the mother-infant relationship at the nursery. Infant Mental Health $J$ 2003; 24(2): 93-110.

14 Seng J, Low L, Sperlich M, Ronis D, Liberzon I. Post-traumatic stress disorder, child abuse history, birthweight and gestational age: a prospective cohort study. BJOG 2011; 118(11): 1329-1339.

15 Keim S, Daniels J, Dole N, Herring A, Siega-Riz A, Scheidt P. A prospective study of maternal anxiety, perceived stress, and depressive symptoms in relation to infant cognitive development. Early Hum Dev 2011; 87(5): 373-380.

16 Forcada-Guex M, Pierrehumbert B, Borghini A, Moessinger A, Muller-Nix C. Early dyadic patterns of mother-infant interactions and outcomes of prematurity at 18 months. Pediatrics 2006; 118(1): e107-e114.

17 Kazak A. Pediatric psychosocial preventative health model (PPPHM): research, practice, and collaboration in pediatric family system medicine. Fam Syst Health 2006; 24(4): 381-395.

18 Bonanno GA, Westphal M, Anthony D, Mancini AD. Resilience to loss and potential trauma. Ann Rev Clin Psychol 2011; 7: 511-535.

19 Kim WJ, Lee E, Namkoong K, Park ES, Rha D-W. Progress of PTSD symptoms following birth: a prospective study in mothers of high-risk infants. $J$ Perinatol 2015; 35(8): 575-579. 
20 Segre L, O'Hara M, Arndt S, Tatano-Beck C. Nursing care for postpartum depression, part 1: do nurses think they should offer both screening and counseling? Am J Matern Child Nurs 2010; 35(4): 220-225.

21 Segre L, Chuffo-Siewert R, Brock R, O'Hara M. Emotional distress in mothers of preterm hospitalized infants: a feasibility trial of nurse-delivered treatment. J Perinatol 2013; 33(12): 924-928.

22 Segre L, Brock, O'Hara M. Depression treatment for impoverished mothers by point-of-care providers: a randomized controlled trial. J Consult Clin Psychol 2015 83(2): 4-324.

23 Roman L, Lindsay J, Boger R, DeWys M, Beaumont E, Jones A et al. Parent-toparent support initiated in the neonatal intensive care unit. Res Nurs Health 1995, 18(5): 385-394.

24 Preyde M, Ardal F. Effectiveness of a parent 'buddy' program for mothers of very preterm infants in a neonatal intensive care unit. CMAJ 2003; 168(8): 969-973.

25 Lindsay J, Roman L, DeWys M, Eager M, Levick J, Quinn M. Creative caring in the NICU: parent-to-parent support. Neonatal Netw 1993; 12(4): 37-44.

26 Goodman J. Women's attitudes, preferences and perceived barriers to treatment for perinatal depression. Birth 2009; 36(1): 60-69.

27 Sword W, Busser D, Ganann R, McMillan T, Swinton M. Women's care-seeking experiences after referral for postpartum depression. Qual Health Res 2008; 18(9): 1161-1173.

28 Dennis C, Chung-Lee L. Postpartum depression help-seeking barriers and maternal treatment preference: a qualitative systematic review. Birth 2006; 33(4): 323-331.

29 Jotzo M, Poets $C$. Helping parents cope with the trauma of premature birth: an evaluation of a trauma-preventive psychological intervention. Pediatrics 2005; 115 (4): 915-919.

30 Shaw R, St John N, Lilo E, Jo B, Benitez W, Stevenson D et al. Prevention of traumatic stress in mothers of preterm infants: a randomized controlled trial. Pediatrics 2013; 132(4): 1-9.

31 Shaw R, Sweetster C, St John N, Lilo E, Corcoran J, Jo B et al. Prevention of postpartum traumatic stress in mothers with preterm infants: manual development and evaluation. Issues Ment Health Nurs 2013; 34(8): 578-586.

32 Shaw R, St John N, Lilo E, Jo B, Benitez W, Stevenson D et al. Prevention of traumatic stress in mothers of preterms: 6-month outcomes. Pediatrics 2014; 134 e481-e488.

33 Zanardo V, Gambina I, Begley C, Litta P, Cosmi E, Giustardi A et al. Psychological distress and early lactation performance in mothers of late preterm infants. Early Hum Dev 2011; 87(4): 321-323.

34 Gjerdingen D, Crow S, McGovern P, Miner M, Center B. Postpartum depression screening at well-child visits: validity of a 2-question screen and the PHQ-9. Ann Fam Med 2009; 7(1): 63-70.

35 Prins A, Ouimette P, Kimerling R, Cameron R, Hugelshofer D, Shaw-Hegwer J et al. The primary care PTSD screen (PC-PTSD): development and operating characteristics. Prim Care Psychiatry 2003; 9(1): 9-14.

36 Beck A, Ward C, Mendelson M, Mock J, Erbaugh J. An inventory for measuring depression. Arch Gen Psychiatry 1961; 4(6): 561-571.

37 Radloff L. The CES-D Scale: a self-report depression scale for research in the general population. Appl Psychol Meas 1977; 1(3): 358-401.

38 Cox J, Holden J, Sagovsky R. Detection of postnatal depression: development of the 10-item Edinburgh Postnatal Depression Scale. Br J Psychiatry 1987; 150(6): 782-786.

39 Eberhard-Gran M, Eskild A, Tambs K, Opjordsmoens S, Samuelsen S. Review of validation studies of the Edinburgh Postnatal Depression Scale. Acta Psychiatr Scand 2001; 104(4): 243-249.

40 Beck C, Gable R. Postpartum Depression Screening Scale: development and psychometric testing. Nurs Res 2000; 49(5): 272-282.

41 Davidson J, Book S, Colket J, Tupler L, Roth S, David D et al. Assessment of a new self-rating scale for post-traumatic stress disorder. Psychol Med 1997; 27(1): 153-160.

42 Callahan J, Borja S, Hynan M. Modification of the Perinatal PTDS Questionnaire to enhance clinical utility. J Perinatol 2006; 26(9): 533-539.
43 DeMier R, Hynan M, Harris H, Manniello R. Perinatal stressors as predictors of symptoms of posttraumatic stress in mothers of infants at high- risk. J Perinatol 1996; 16(4): 276-280.

44 Sampilo M, Lassen S, Belmont J, Parimi P. Development and validation of a psychosocial screening tool to identify high-risk families in the neonatal intensive care unit. The PAT-NICU. Poster Presented at the Annual Conference of the Society of Pediatric Psychology, San Antonio, TX, USA, 2011.

45 Meyer E, Zeanah C, Boukydis C, Lester B. A clinical interview for parents of high-risk infants: concept and applications. Infant Mental Health J 1993; 14(3): 192-207.

46 Mounts K. Screening for maternal depression in the neonatal ICU. Clin Perinato 2009; 36(1): 137-152.

47 De Figueiredo F, Parada A, Cardoso V, Batista R, da Silva A, Barbieri M et al Postpartum depression screening by telephone: a good alternative for public health and research. Arch Womens Ment Health 2014; 18(3): 547-553.

48 Dennis $C$. The process of developing and implementing a telephone-based peer support program for postpartum depression: evidence from two randomized controlled trials. Trials 2014; 15: 131.

49 Fortney J, Pyne J, Kimbrell T, Hudson T, Robinson D, Schneider R et al. Telemedicine-based collaborative care for posttraumatic stress disorder: a randomized clinical trial. JAMA Psychiatry 2015; 72(1): 58-67.

50 Shore J. Telepsychiatry: videoconferencing in the delivery of psychiatric care. Am J Psychiatry 2013; 170(3): 256-262.

51 National Perinatal Association's Family Advocacy Network. Available at: www. nationalperinatal.org/parentsupportgroups (date last accessed 13 August 2015).

52 Preemie Parent Alliance. Available at: http://preemieparentalliance.weebly. com/\#/ (date last accessed 13 October 2015).

53 The March of Dimes. Available at: www.shareyourstory.org (date last accessed 13 October 2015).

54 The European Foundation for the Care of Newborn Infants. Available at: www. efcni.org (date last accessed 13 October 2015).

55 The Canadian Premature Babies Foundation. Available at: www.cpbf-fbpc.org (date last accessed 13 October 2015).

56 Postpartum Support International. Available at. www.postpartum.net (date last accessed 13 October 2015)

57 Johansson R, Andersson G. Internet-based psychological treatments for depression. Expert Rev Neurother 2012; 12(7): 861-870.

58 Khatri N, Marziali E, Tchernikov I, Shepherd N. Comparing telehealth-based and clinic-based group cognitive behavioral therapy for adults with depression and anxiety: a pilot study. Clin Interv Aging 2014; 9: 765-770.

59 Meltzer-Brody S, Stuebe A. The long-term psychiatric and medical prognosis of perinatal mental illness. Best Pract Res Clin Obstet Gynaecol 2014; 28(1): 49-60.

60 Brandon A, Trivedi M, Hynan L, Miltenberger P, Labat D, Rifkin J, Stringer C. Prenatal depression in women hospitalized for obstetric risk. J Clin Psychiatry 2008; 69(4): 635-643.

61 Grigoriadis S, vonderporten E, Mamisashvilli L, Tomlinson G, Dennis C, Koren G et al. The impact of maternal depression during pregnancy on perinatal outcomes: a systematic review and meta-analysis. J Clin Psychiatry 2013; 74(1): e321-e341.

62 Shaw J, Asch S, Kimerling R, Frayne S, Shaw K, Phibbs C. Posttraumatic stress disorder and risk of spontaneous preterm birth. Obstet Gynecol 2014; 124(6): 1111-1119.

63 Yonkers K, Smith M, Forray A, Epperson C, Costello D, Lin H et al. Pregnant women with posttraumatic stress disorder and risk of preterm birth. JAMA Psychiatry 2014 71(8): 897-904.

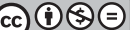

This work is licensed under a Creative Commons AttributionNonCommercial-NoDerivs 4.0 International License. The images or other third party material in this article are included in the article's Creative Commons license, unless indicated otherwise in the credit line; if the material is not included under the Creative Commons license, users will need to obtain permission from the license holder to reproduce the material. To view a copy of this license, visit http:// creativecommons.org/licenses/by-nc-nd/4.0/ 